\title{
Nivel de desarrollo cognitivo y de habilidades motrices básicas en escolares de diferentes sexos
}

\section{Level of cognitive development and basic motor skills in schoolchildren of}

\section{different sexes}

1 Luis Alfredo Jiménez Ruiz $\quad$ (iD) https://orcid.org/0000-0001-8704-0563 Universidad Técnica de Ambato, Facultad de Ciencias de la Educación, Carrera de Pedagogía de la Actividad Física y Deporte. Ambato, Ecuador, la.jimenez@uta.edu.ec

2 Jimmy Eduardo Sánchez Coca

(iD) https://orcid.org/0000-0002-4424-1290 Universidad Técnica de Ambato, Facultad de Ciencias de la Educación, Carrera de Pedagogía de la Actividad Física y Deporte. Ambato, Ecuador, jsanchez4565@uta.edu.ec

3 Dennis José Hidalgo Alava https://orcid.org/0000-0002-2234-5072 Universidad Técnica de Ambato, Facultad de Ciencias de la Educación, Carrera de Pedagogía de la Actividad Física y Deporte. Ambato, Ecuador dj.hidalgo@uta.edu.ec

4 Christian Mauricio Sánchez Cañizares (iD) https://orcid.org/0000-0002-0677-7246 Universidad Técnica de Ambato, Facultad de Ciencias de la Educación, Carrera de Pedagogía de la Actividad Física y Deporte. Ambato, Ecuador cm.sanchezc@uta.edu.ec

Artículo de Investigación Científica y Tecnológica Enviado: 24/12/2021

Revisado: 29/12/2021

Aceptado: $12 / 01 / 2022$

Publicado:08/03/2023

DOI: https://doi.org/10.33262/concienciadigital.v6i1.4.2060

Cítese:

Jiménez Ruiz, L. A., Sánchez Coca, J. E., Hidalgo Alava, D. J., \& Sánchez Cañizares, C. M. (2023). Nivel de desarrollo cognitivo y de habilidades motrices básicas en escolares de diferentes sexos. ConcienciaDigital, 6(1.4), 1157-1169. https://doi.org/10.33262/concienciadigital.v6i1.4.2060

CONCIENCIA DIGITAL, es una Revista Multidisciplinar, Trimestral, que se publicará en soporte electrónico tiene como misión contribuir a la formación de profesionales competentes con visión humanística y crítica que sean capaces de exponer sus resultados investigativos y científicos en la misma medida que se promueva mediante su intervención cambios positivos en la sociedad. https://concienciadigital.org

La revista es editada por la Editorial Ciencia Digital (Editorial de prestigio registrada en la Cámara Ecuatoriana de Libro con No de Afiliación 663) www.celibro.org.ec 
Palabras claves: desarrollo cognitivo, educación, habilidad motriz, pensamiento

Keywords: cognitive development, education, motor skills, thinking.
Resumen

En edades de iniciación escolar sin importancia del sexo, el desarrollo cognitivo no limita el nivel de habilidades motrices básica como son sentido de equilibrio, la locomoción y la manipulación. Objetivo: En el presente trabajo investigativo se analizó la relación existente entre el nivel de desarrollo cognitivo y el nivel de las habilidades motrices básicas en escolares de la Unidad Educativa Oxford de la ciudad de Ambato, durante el periodo abril-agosto de 2021. Metodología: Se trata de una investigación cuantitativa, no experimental de alcance correlacional con método un método de investigación hipotéticodeductivo. Para la recolección de datos se utilizaron dos instrumentos que permitieron medir cada una de las variables en una muestra de estudio de 18 escolares perteneciente a la Unidad educativa de la ciudad Ambato. Resultados: Los resultados de la investigación fueron comprobados a través de la prueba Chicuadrado con diferenciación asintótica bilateral en un nivel de $\mathrm{P} \geq 0.05$ dando como resultado un valor estadístico no significativo en el nivel de relación entre las variables estudiadas. Conclusión: Se concluye que el nivel de desarrollo cognitivo en estas edades sin importancia del sexo del escolar no se relaciona con el nivel de habilidades motrices básicas.

\section{Abstract}

In school initiation ages regardless of sex, cognitive development does not limit the level of basic motor skills such as a sense of balance, locomotion, and manipulation. Objective: In this research work, the relationship between the level of cognitive development and the level of basic motor skills in schoolchildren of the Oxford Educational Unit of the city of Ambato, during the period April-August 2021, was analyzed. Methodology: It is a quantitative, non-experimental investigation of correlational scope with a hypothetical-deductive method of investigation. For data collection, two instruments were used that made it possible to measure each of the variables in a study sample of 18 schoolchildren belonging to the Educational Unit of the city of Ambato. Results: The research results were verified through the Chi-square test with bilateral asymptotic differentiation at a level of $\mathrm{P} \geq 0.05$, resulting in a non-significant statistical value at the level of relationship between the variables studied. Conclusion: 
It is concluded that the level of cognitive development in these ages regardless of the school's gender is not related to the level of basic motor skills.

\section{Introducción}

La cognición es un proceso del ser humano que contiene muchos factores en los que están implicados procesos como el pensamiento, la capacidad lingüística, lo sensorial, las percepciones, la función mnésica, atención, razonamiento, pensamiento crítico y la resolución de conflictos, para evaluar diferentes situaciones y se realice una correcta toma de decisiones, dando como resultado el desarrollo intelectual. Las capacidades cognitivas se encuentran relacionado con la motricidad, mediante la cognición ya que ayuda al desarrollo y perfeccionamiento de los patrones motrices, por lo cual es importante determinar la incidencia entre el desarrollo cognitivo y el nivel de las habilidades motrices básicas en escolares.

Revisando la literatura, un buen desarrollo cognitivo depende de varios factores ambientales. Carrero (2018), menciona una buena nutrición y hábitos de vida saludable, ayuda a un buen desempeño en lo académico tanto en cognitivo como lo motriz, una mala nutrición puede causar problemas de salud que afectan el desarrollo cognitivo del niño.

La estimulación sensorial es fundamental ya que ayuda al desarrollo cognitivo, incidiendo en el rendimiento académico, en clases de educación física es importante el desarrollo cognitivo ya que ayuda a la coordinación y a la armonía de sus movimientos.

Las habilidades motrices según Hsiao (2016), son primordiales en edad escolar, también menciona que las habilidades motrices tienen relación con conocer su cuerpo para realizar el movimiento y lograrlo adecuadamente, la clasificación de las habilidades motrices es la estabilidad, la locomoción y la manipulación, el objetivo de estas áreas es causar una mejora en la capacidad de ejercicio como también en su crecimiento. En los escolares para consolidar las habilidades motoras según Tomac (2012), en necesario crear memoria muscular en base a repeticiones que desarrolla una estabilidad y precisión de las habilidades motoras, el desarrollo evolutivo de la motricidad inicia con la inhibición de los reflejos que dan lugar a movimientos bruscos sin coordinación en los primeros meses de vida, en la segunda infancia se desarrolló la motricidad fina y gruesa que permite la coordinación de los movimientos y la interacción con el medio ambiente para el desplazamiento, a los 6 años se da le perfeccionamiento en la motricidad gruesa siendo capas de camina, correr, saltar y la motricidad fina culmina con el perfeccionamiento del proceso de la escritura. El conocimiento de las etapas evolutivas del desarrollo motor es importante en el contexto educativo, ya que permite la potenciación correcta en los 
estudiantes (Nazario, 2014), el proceso de enseñanza y el aprendizaje durante las clases de Educación Física deben potenciar el desarrollo motor en los niños (Bonilla et al., 2018).

Las habilidades motrices básicas según Altinkök (2016), se desarrolla en los estudiantes de una mejor forma cuando se emplea actividades recreativas, ya que ellos se imaginan su forma de moverse e interactuar con los demás, las habilidades motrices básicas van de la mano con el aprendizaje del diario vivir, por lo cual es importante mantener el perfil del estudiante y educar acorde a su edad para tener un aprendizaje significativo y un mejor rendimiento motriz. Como refiere Araujo (2012), la aplicación de actividades físicas y deportivas fomenta el desarrollo de habilidades motrices básicas, el realizar actividad física o deporte evita el sedentarismo que ayuda al desarrollo del niño evitando la obesidad infantil, las clases de educación física aportar a la salud del estudiante y al perfeccionamiento de las habilidades motrices básicas.

El desarrollo cognitivo y la motricidad desde el punto de vista de Wu (2017), están estrechamente ligados el retraso en la cognición afecta a los patrones motrices básicos, la alteración de las capacidades cognitivas afectan a la velocidad del pensamiento, concentración y memoria que están ligados en la ejecución correcta de los movimientos, un ejemplo es cuando un niño nace prematuro a sus 11 años su habilidad cognitiva es más lenta con una comorbilidad en el retraso del desarrollo motor que la de una niño que nación de forma normal, este suceso da como resultado que los niños nacidos prematuramente desarrollan un problema de habilidades cognitivas, siendo importante realizar un diagnóstico a edad temprana de las capacidades cognitivas del niño para poder subsanar retrasos que se puedan presentar a nivel motriz. El desarrollo de la capacidad cognitivas adecuadas es necesaria para evitar problemas en el desempeño académico que puede generar un bajo rendimiento, como menciona Mego (2021) las habilidades cognitivas se encuentran en todo momento y en cada una de las facetas de la vida de cada persona y axiomas que se presentan en cada experiencia que ha vivido el estudiante, el impacto que causan las habilidades cognitivas en los estudiantes es que se desarrollan paso a paso en cada uno de los procesos de aprendizaje el cual el educando aprende a reconocer y relacionar, inferir y deducir, generalizar y elaborar dando como resultado un buen desenvolvimiento y cumplir con el currículo institucional, en especial en las clases de cultura física en la que se da la integración del pensamiento con la ejecución adecuada de los movimientos.

El correcto empleo del método pedagógico es importante en el proceso cognitivo ya que este permite la correcta adquisición almacenamiento y codificación de la información generando un aprendizaje significativo (Prokhorov, 2019). El aporte de técnicas pedagógicas en el proceso académico permite la eficacia en el aprendizaje de los estudiantes, la formación de métodos bien estructurados es importantes para la enseñanza 
que da como resultado un proceso cognitivo favorable para los educandos. Las acciones mentales organizadas favorecen al entorno en el que se está educando al estudiante, facilitando la integración y desarrollo de la motricidad especialmente en las clases de cultura física. Según Pereira (2019), los procesos cognitivos de los estudiantes se fomentan en las clases de educación física ya que ayuda al desarrollo del pensamiento y la imaginación, debido al aumento del nivel de concentración al recibir instrucciones del docente para ejecutar las actividades prácticas, en la motivación ya que involucra la integración del pensamiento con los movimientos para lograr los objetivos en la clase.

Es importante analizar la relación del desarrollo cognitivo y el nivel de las habilidades motrices en escolares, ya que aporta en la enseñanza beneficios de aplicar lo cognitivo con lo motriz en los estudiantes. Que permitirá mejor el aprendizaje y el rendimiento académico, permitiendo generar un método adecuado de enseñanza.

\section{Metodología}

Se trata de una investigación con enfoque cuantitativo porque basado en un análisis estadístico de los datos recopilados. Los instrumentos de recolección de datos se plantearon con base en los objetivos propuestos y según los resultados se pudo verificar una de las dos hipótesis planteadas. Al no experimentar con la población, es decir no hubo intervención por parte del investigador, la investigación es considerada no experimental. Se analizaron datos de las variables de estudio en un tiempo determinado sobre la población por lo que es de tipo transversal y correlacional ya que se midió el nivel de relación entre las dos variables. El desarrollo de la investigación se estableció aplicando el método hipotético-deductivo.

La población de estudio fueron estudiantes de la unidad educativa Oxford de la ciudad de Ambato, de la que se estableció como muestra de 18 estudiantes, 11 hombres y 7 mujeres con edades comprendidas entre los 10 y 11 años.

Para conocer el nivel del desarrollo cognitivo se aplicó la prueba de la figura humana el cual mediante la interpretación de los dibujos de los estudiantes se procedió a la evaluación, la cual consistió en verificar las habilidades intelectuales de los educandos como también el estado emocional

Para medir el nivel de habilidades motrices básicas se aplicó una batería para niños de entre 5 y 11 años, la que permite conocer el estado de motricidad, mediante la observación, este permite analizar los diferentes tipos de habilidades motrices mediante la ejecución de ejercicios específicos, calificados a posterior en base a baremos estandarizados para determinar el estado de habilidad motriz de cada uno de los estudiantes (Palacio, 2021). 
El tratamiento estadístico de los datos y resultados de la investigación se realizó a través del software estadístico SPSS versión 25 IBM para Windows. De las variables cualitativas se analizaron frecuencias, porcentajes y se efectuó una interpretación de tipo descriptiva tanto en la caracterización de la muestra de estudio, así como en los resultados generales. En el análisis general de significación en primer lugar se aplicó una prueba de normalidad de Shapiro - Wilk, que determino la aplicación de la prueba no paramétrica U de Mann Withney para muestras independientes y la prueba Chi-cuadrado de Pearson para variables cualitativas que permitió determinar la relación entre los niveles de las variables de estudio.

\section{Resultados}

Los resultados se encuentran representados en tablas y en concordancia a los objetivos planteados.

\section{Caracterización de la muestra de estudio}

En la tabla 1, se visualizan la distribución frecuencial y porcentual por sexo y los valores descriptivos de la edad.

\section{Tabla 1}

Caracterización de la muestra de estudio

\begin{tabular}{|c|c|c|c|c|c|c|c|}
\hline \multirow{2}{*}{ Sexo } & \multirow{2}{*}{$\mathrm{f}$} & \multirow{2}{*}{$\%$} & \multicolumn{5}{|c|}{ Edad } \\
\hline & & & Mínimo & Máximo & Media & DS & $\mathrm{P}$ \\
\hline Masculino & 11 & 61.1 & 10 & 11 & 10.64 & \pm 0.51 & \\
\hline Femenino & 7 & 38.9 & 10 & 11 & 10.43 & \pm 0.54 & $0.401 * *$ \\
\hline Total & 18 & 100.0 & 10 & 11 & 10.56 & \pm 0.51 & \\
\hline
\end{tabular}

El sexo masculino presenta un mayor porcentaje de contribución a la muestra de estudio en 4 integrantes más a diferencia del sexo femenino, en relación con la edad media de los mismos la diferencia es mínima con 0.21 años a favor del sexo masculino y sin diferencias significativas en un nivel de $\mathrm{P} \geq 0.05$ entre los grupos por sexo que determinan la igualdad de toda la muestra.

Aplicando la metodología propuesta para la investigación se valoró el nivel de desarrollo cognitivo por grupos de sexo, partiendo del coeficiente obtenido en el desarrollo de la prueba y categorizándolos en los niveles establecidos en la tabla 2. 


\section{Tabla 2}

Nivel de desarrollo cognitivo

\begin{tabular}{|c|c|c|c|c|c|c|c|c|c|c|}
\hline \multirow{3}{*}{$\begin{array}{l}\text { Niveles de } \\
\text { desarrollo } \\
\text { cognitivo }\end{array}$} & \multicolumn{10}{|c|}{ Sexo } \\
\hline & \multicolumn{3}{|c|}{ Masculino } & \multicolumn{3}{|c|}{ Femenino } & \multirow{2}{*}{$\mathrm{P}$} & \multicolumn{3}{|c|}{ Total } \\
\hline & $\mathrm{f}$ & $\%$ & M CDC & $\mathrm{f}$ & $\%$ & M CDC & & $\mathrm{f}$ & $\%$ & M CDC \\
\hline $\begin{array}{c}\text { Normal alto a } \\
\text { superior }(>110)\end{array}$ & 11 & 100.0 & & 3 & 42.9 & & & 14 & 77.8 & \\
\hline $\begin{array}{l}\text { Normal a normal- } \\
\text { alto }(85-120)\end{array}$ & 0 & 0.00 & 7.55 & 4 & 57.1 & 6.14 & $0.04 *$ & 4 & 22.2 & 7 \\
\hline Total & 11 & 100 & & 7 & 100.0 & & & 18 & 100.0 & \\
\hline
\end{tabular}

Los resultados obtenidos evidenciaron que en relación con el sexo masculino en su totalidad los estudiantes se encontraron en un nivel normal alto a superior y en el sexo femenino existió una distribución con mayor frecuencia en un nivel normal a normal alto. Los valores medios del coeficiente de desarrollo cognitivo (CDC) presentaron una diferencia de 1.41 puntos a favor del sexo masculino con diferencias significativas en un nivel de $\mathrm{P}<0.05$ entre los grupos por sexo.

Para la evaluación del nivel de desarrollo de habilidades motrices básicas, en primer lugar, se valoraron los índices de desarrollo habilidades motrices básicas de locomoción, manipulación y estabilidad y a posterior se calculó el coeficiente de desarrollo de habilidades motrices básicas tabla 3 .

Tabla 3

Habilidades motrices básicas

\begin{tabular}{cccccccc}
\hline \multirow{2}{*}{ Parámetros } & \multicolumn{7}{c}{ Sexo } \\
\cline { 2 - 7 } & \multicolumn{2}{c}{ Masculino } & \multicolumn{2}{c}{ Femenino } & \multicolumn{2}{c}{ Total } & P \\
\cline { 2 - 7 } & M & DS & M & DS & M & DS & $0.670^{* *}$ \\
\hline IHMBL & 23.91 & 1.044 & 23.57 & 1.512 & 23.78 & 1.215 & $0.889^{* *}$ \\
\hline IHMBM & 35.91 & 1.578 & 36.00 & 1.528 & 35.94 & 1.514 & $0.881^{* *}$ \\
\hline IHMBE & 13.55 & 1.036 & 13.71 & 1.113 & 13.61 & 1.037 & $0.336^{* *}$ \\
\hline CHMB & 73.36 & 1.963 & 73.29 & 2.812 & 73.33 & 2.249 & \\
\hline
\end{tabular}

Los resultados alcanzados en relación a sus valores medios (M) y desviaciones estándares (DS), descriptivamente evidenciaron una diferencia mínima a favor del sexo masculino 
en relación al índice de desarrollo de habilidades motrices básicas de locomoción (IHMBL) en 0.13 puntos, en relación al índice de desarrollo de habilidades motrices básicas de manipulación (IHMBM) el sexo femenino presento 0.03 puntos superior al igual que en el índice de desarrollo de habilidades motrices básicas de estabilidad con 0.06 puntos. En relación con el coeficiente de desarrollo de habilidades motrices básicas (CHMB) el sexo masculino presento 0.03 puntos a favor, no obstante, tanto a nivel de los índices parciales y el coeficiente general calculado, no existen diferencias significativas entre grupos con un valor de $\mathrm{P} \geq 0.05$, que determina una igualdad de resultados.

Los resultados obtenidos permitieron categorizar a la muestra de estudio en diferentes niveles según los baremos estándares para la prueba escogido, como muestra la tabla 4.

\section{Tabla 4}

Niveles de habilidades motrices básicas

\begin{tabular}{cccccccc}
\hline \multirow{2}{*}{$\begin{array}{c}\text { Niveles de } \\
\text { desarrollo de } \\
\text { habilidades } \\
\text { motrices básicas }\end{array}$} & \multicolumn{2}{c}{ Masculino } & \multicolumn{2}{c}{ Femenino } & \multicolumn{2}{c}{ Total } & P \\
\cline { 2 - 7 } & $\mathrm{f}$ & $\%$ & $\mathrm{f}$ & $\%$ & $\mathrm{f}$ & $\%$ \\
\hline Bajo & 2 & 18.2 & 2 & 28.6 & 4 & 22.2 \\
Medio & 2 & 18.2 & 0 & 0 & 2 & 11.1 & 0.468 \\
Alto & 7 & 63.6 & 5 & 71.4 & 12 & 66.7 & 100.0 \\
\hline Total & 11 & 100.0 & 7 & 100.0 & 18 &
\end{tabular}

El análisis de distribución por niveles evidencio que en ambos sexos existió una predominancia del nivel alto de desarrollo cognitivo con más del 50\% en cada grupo, estadísticamente no existieron diferencias significativas entre los grupos con un valor de $\mathrm{P} \geq 0.05$, determinando que al igual que los coeficientes el desarrollo es igualitario en ambos sexos.

Con los resultados obtenidos de cada variable y sus respectivos niveles se procedió al análisis de relación entre las dos variables a nivel descriptivo y estadístico, como se muestra en la tabla 5 . 


\section{Tabla 5}

Resultados de análisis de la relación entre las dos variables

\begin{tabular}{|c|c|c|c|c|c|c|}
\hline \multirow{3}{*}{ Sexo } & \multirow{3}{*}{$\begin{array}{l}\text { Variables de } \\
\text { estudio }\end{array}$} & \multirow{3}{*}{ Niveles } & \multicolumn{4}{|c|}{ Nivel desarrollo de habilidades motrices } \\
\hline & & & & básicas & & Total \\
\hline & & & Bajo & Medio & Alto & \\
\hline \multirow[t]{2}{*}{ Masculino } & $\begin{array}{l}\text { Desarrollo } \\
\text { cognitivo }\end{array}$ & $\begin{array}{c}\text { Normal alto a superior } \\
\qquad(>110)\end{array}$ & 2 & 2 & 7 & 11 \\
\hline & & Total & 2 & 2 & 7 & 11 \\
\hline \multicolumn{3}{|c|}{$\mathrm{P}$} & \multicolumn{4}{|c|}{$\begin{array}{l}\text { No aplica la variable de desarrollo cognitivo es } \\
\text { constante }\end{array}$} \\
\hline \multirow{3}{*}{ Femenino } & \multirow{2}{*}{$\begin{array}{l}\text { Desarrollo } \\
\text { cognitivo }\end{array}$} & $\begin{array}{c}\text { Normal a normal-alto } \\
(85-120)\end{array}$ & 2 & 0 & 2 & 4 \\
\hline & & $\begin{array}{l}\text { Normal alto a superior } \\
\qquad(>110)\end{array}$ & 0 & 0 & 3 & 3 \\
\hline & & Total & 2 & 0 & 5 & 7 \\
\hline \multicolumn{3}{|c|}{$\mathrm{P}$} & \multicolumn{4}{|c|}{0.147} \\
\hline \multirow{3}{*}{ Total } & \multirow{3}{*}{$\begin{array}{l}\text { Desarrollo } \\
\text { cognitivo }\end{array}$} & $\begin{array}{c}\text { Normal a normal-alto } \\
(85-120)\end{array}$ & 2 & 0 & 2 & 4 \\
\hline & & $\begin{array}{l}\text { Normal alto a superior } \\
\qquad(>110)\end{array}$ & 2 & 2 & 10 & 14 \\
\hline & & Total & 4 & 2 & 12 & 18 \\
\hline \multicolumn{3}{|c|}{$P$} & \multicolumn{4}{|c|}{0.276} \\
\hline
\end{tabular}

En el sexo masculino no se pudo aplicar por la constante en el nivel de desarrollo cognitivo, sin embargo, se evidencio que en relación con el nivel de desarrollo de habilidades motrices básicas existió una distribución en los 3 niveles establecidos con tendencia al nivel alto. En el sexo femenino los escolares con un nivel normal a normalalto de desarrollo cognitivo se distribuyeron en niveles bajos y altos de habilidades motrices básicas y en el nivel de normal alto a superior todos se encontraron en un nivel alto de habilidades motrices básicas, fenómeno similar en relación con la muestra total de estudio. Estadísticamente en ninguno de los grupos por sexo y a nivel general se encontraron diferencias significativas con un valor de $\mathrm{P} \geq 0.05$, determinando que no existe una relación directa entre el nivel de desarrollo cognitivo y de desarrollo de habilidades motrices básicas y que esta hipótesis no se diferencia en relación con el sexo del estudiante.

\section{Discusión}

Los resultados de la presente investigación han permitido la evaluación de las habilidades motrices básicas en escolares se utilizó una batería de habilidades motrices para evaluar el estado motriz de los participantes, a comparación de Burgos (2017), quien menciona 
que para evaluar las HMB utilizo una encuesta a los maestros para conocer si ellos disponen del tiempo para poder utilizar métodos didácticos en la enseñanza y desarrollo de habilidades motrices, por otro lado se realizó otra encuesta a los representantes de cada niño para conocer su nivel de desarrollo motriz. Aporta Roa (2019), diciendo que para el desarrollo y evaluación de HMB el utilizo un método combinado el cual realizo una serie de actividades motrices las cuales se las fueron realizando por sesiones conjunto a los padres de cada niño y se aplicó un baremo de (bien, regular, mal,), para verificar el nivel de desarrollo de HMB de los niños, esta tabla se aplicó en cada prueba de habilidad. Menciona Rueda (2017), que para el desarrollo de las motricidad y cognitivo utilizo el test de visual-motor Bender-Gestalt el cual tiene un sistema de puntuación gradual denominado B-SPG (Adolph, 2017), esto se utiliza para evaluar el nivel perceptivo-motor y se aplica desde los 6 hasta los 10 años a través de gráficos, en comparación con mis instrumentos de evaluación los cuales fueron por separado y el test de la figura humana es similar a este test por que se apoyan en gráficos, para mi evaluación se utilizó puntuaciones y un baremo para conocer su nivel tanto motriz como cognitivo, comparando con este autor utilizo una puntuación gradual donde cero puntos cuando no hay errores, por lo tanto 1,2 y 3 indican problemas mayores en la calidad del dibujo.

Manifiesta Carcamo-Oyarzun (2020), que para evaluar las habilidades motrices básicas utilizo la batería de MOBAK 5-6 la cual consiste en conocer el estado de habilidad motriz básica de cada niño a evaluar al igual que mi investigación este autor también trabajo con 6to grado, esta batería consta de parámetros a evaluar que son atrapar, lanzar, conducir el balón con la mano al igual que con el pie y tiene una puntuación de 0 a 2 aciertos 0 puntos, de 3 a 4 aciertos $=1$ punto y de 5 a 6 aciertos $=2$ puntos esto aplica para todas las pruebas, comparando con mi evaluación de motricidad no tiene similitud ya que el instrumento que utilice tiene métodos diferentes de calificación y pruebas mucho más complejas donde sí se puede saber el nivel de motricidad básica que tiene cada escolar.

\section{Conclusiones}

- Después de haber realizado la respectiva evaluación y obtener los resultados se llegaron a las siguientes conclusiones.

- La valoración del nivel de desarrollo cognitivo en ambos sexos determino que el grupo masculino se encentraban en un mismo nivel (normal alto a superior), a diferencia del grupo de sexo femenino que presento una distribución en ambos niveles con prevalencia en el normal alto a superior.

- La evaluación del desarrollo de habilidades motrices básicas determino que, tanto en el sexo masculino como femenino en relación con índices y coeficientes, son grupos homogéneos con una diferencia mínima descriptiva y la distribución niveles evidencio una prevalencia hacia el nivel alto. 
- El análisis de la relación entre niveles fue negativa, estadísticamente sustentadas en la ausencia de diferencias significativas por grupos de sexo y entre niveles por grupos, determinando que, en estas edades sin importar el sexo del escolar, el nivel de desarrollo cognitivo no incide en el nivel de desarrollo de habilidades motrices básicas.

\section{Referencias Bibliográficas}

Adolph, K. E. (2017). Motor development. Handbook of child psychology, 2.

Altinkök, M. (2016). he Effects of Coordination and Movement Education on Pre School Children's Basic Motor Skills Improvement. Universal Journal of Educational Research, 4(5), 1050-1058.

Araujo, M. P. (2012). Contribution of different contents of physical education classes in elementary school I for the development of basic motor skills. Revista Brasileira de Medicina do Esporte, 18, 153-157.

Bonilla, J. A. M., Ortiz, P. G. O., Zapata, E. G., \& Zúñiga, A. V. C. (2018). Efectos de un programa de intervención en la motricidad gruesa: estudio con niños de 5 a 7 años. Ciencia Digital, 2(2), 64-78.

Burgos Ángulo, D. J. (2017). Estrategia didáctica para el desarrollo de las habilidades motrices básicas en Educación Física del subnivel básico elemental (Bachelor's tesis, Universidad de Guayaquil, Facultad de Educación Física, Deportes y Recreación).

Carcamo-Oyarzun, J. \&. (2020). Validez de constructo de la batería MOBAK para la evaluación de las competencias motrices básicas en escolares de educación primaria. Revista Española de Pedagogía, 78(276), 291-308.

Carrero, C. M. (2018). Anemia infantil: desarrollo cognitivo y rendimiento académico. Archivos Venezolanos de farmacología y terapéutica, 37(4), 411- 426.

Hsiao, H. S. (2016). Using a gesture interactive game-based learning approach to improve preschool children's learning performance and motor skills. Computers \& Education, vol. 95, p. 151-162.

Mego, C. H. (2021). Las habilidades cognitivas y desarrollo de competencias oral y comprensiva: una revisión bibliográfica. Conrado, 17(78), 189 - 193.

Nazario, P. F. (2014). Revista Brasileira de Cineantropometria \& Desempenho Humano, $16,86-95$. 
Palacio, E. V. (2021). Diseño y validación de una batería de habilidades motrices básicas para niños entre 5 y 11 años. Diseño y validación de una batería de habilidades motrices básicas para niños entre 5 y 11 años., vol. 10, (no 2,), p. 165-181.

Pereira, P. B. (2019). Influence of students' cognitive processes on physical education teachers' thought process. Journal of Physical Education \& Sport, 19.

Prokhorov, A. O. (2019). Interaction of Mental States and Cognitive Processes in Learning Activity of Students. DEGRES, 18(1), 69 - 82.

Roa González, S. V. (2019). Actividades físicas para desarrollar las habilidades motrices básicas en niños del programa Educa a tu Hijo. Conrado, 15(69), 386- 393.

Rueda, F. J. (2017). The Relationship Between Perceptual Motor Skills and Attention1. Paidéia, 27(66), 24-32.

Tomac, Z. H. (2012). The assessment of preschool children's motor skills after familiarization with motor tests. The Journal of Strength \& Conditioning Research, 26(7), 1792-1798.

$\mathrm{Wu}, \mathrm{M}$. L. (2017). Infant motor and cognitive abilities and subsequent executive function. Infant Behavior and Development, 49, 204 - 213.

\section{LCiencia}


El artículo que se publica es de exclusiva responsabilidad de los autores y no necesariamente reflejan el pensamiento de la Revista Conciencia Digital.

\section{Ciencia \\ Ligital}

El artículo queda en propiedad de la revista y, por tanto, su publicación parcial y/o total en otro medio tiene que ser autorizado por el director de la Revista Conciencia Digital.
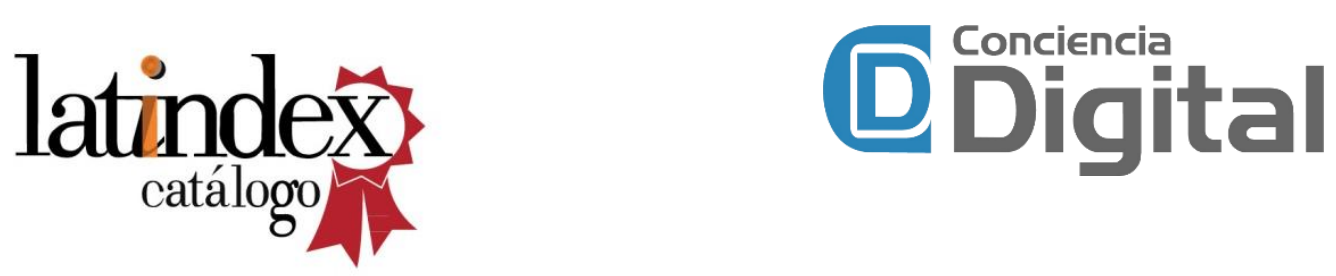

Indexaciones

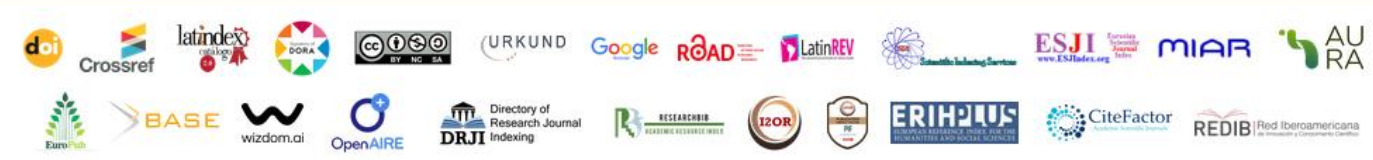

